\title{
On the Performance of Spectrum Sharing Systems with Multiple Antennas
}

\author{
Liang Yang*॰ \\ $*$ Department of Electrical Engineering \\ Jinan University \\ Guangzhou, China
}

\author{
Mohamed-Slim Alouini ${ }^{\dagger}$ \\ $\dagger$ Electrical Engineering Program \\ KAUST \\ Thuwal, Saudi Arabia
}

\author{
Khalid Qaraqe $\diamond$ \\ $\diamond$ Electrical and Computer Engineering Program \\ Texas A\&M University at Qatar \\ Doha, Qatar
}

\begin{abstract}
In this paper, we study the capacity of spectrum sharing (SS) multiple-input multiple-output (MIMO) systems over Rayleigh fading channels. More specifically, we present closed-form capacity formulas for such systems with and without optimal power and rate adaptation. A lower bound on the capacity is also derived to characterize the scaling law of the capacity. Results show that increasing the number of antennas has a negative effect on the system capacity in the low signal-tonoise (SNR) regime and the scaling law at high SNR is similar to the conventional MIMO systems. In addition, a lower bound on the capacity of the SS keyhole MIMO channels is analyzed. We also present a capacity analysis of SS MIMO maximal ratio combining (MRC) systems and the results show that the capacity of such systems always decreases with the increase of the number of antennas. Numerical results are finally given to illustrate our analysis.
\end{abstract}

\section{INTRODUCTION}

The demand of several new wireless communications services is driving the development of new spectrum allocation policies. To satisfy the spectrum demand, cognitive radio (CR) was proposed. The basic idea of CR is to allow the unlicensed users (secondary users) to share the spectrum with the licensed users (primary users) under some power constraints. That is, the interference caused by the secondary users at the primary users should be controlled to an allowable level [1-3]. More specifically, in [2], the authors have considered the channel capacity under received power constraint for different fading channels conditions. In addition, [3] studied the spectrum sharing (SS) systems with multiuser diversity in which one secondary user with the best channel condition is selected for signal transmission.

So far most of the research results for SS systems focused on the single antenna case. In this paper, we extend the results of [2][3] to a multiple-input multiple-output (MIMO) system. For instance, it is well known that applying multiple antennas can provide higher spectrum efficiency for wireless communications [4]. Thus, we check in this paper if MIMO systems can still improve the capacity of SS networks. In particular, we investigate the capacity scaling laws of SS MIMO systems. The contributions of this paper are summarized in what follows.

We analyze single user SS MIMO systems in which the simple spatial multiplexing is used at the secondary transmitters. We offer exact closed-form capacity expressions. Also, a lower bound on the capacity is provided. With the availability of the channel state information (CSI) at the transmitter, optimal power and rate adaptation [5] can be applied. We present in the paper an analysis for such kind of transmission and show that the channel capacity of such kind of schemes is larger than the capacity of systems with uniform power allocation. Next, taking into account a realistic wireless environment, we consider the keyhole effect [6] and we show that the capacity of such channels can be lower bounded by an additive white Gaussian noise (AWGN) channel. Finally, the capacity of a SS network combined with MIMO maximal ratio combing (MRC) transmission [7] is analyzed.

\section{SYSTEM CHANNEL MODELS}

We consider a SS network as shown in Fig.1 where all the nodes are equipped with multiple antennas. For notation simplicity, we assume that all the nodes have the same number of antennas $N$. Let $\mathbf{H}$ and $\mathbf{G}$ denote the channel gain matrices from the secondary transmitter to secondary receiver and the primary receiver, respectively. We assume that the secondary transmitter can obtain the full CSI of the channel matrix $\mathbf{H}$. This can be ensured by using an error-free feedback link. All the entries of $\mathbf{H}$ and $\mathbf{G}$ are independent and identically distributed (i.i.d) complex Gaussian random variables with zero mean and unit variance. To ensure that the interference constraint at the primary receiver is met, we adopt the interference temperature $Q$. From [3], the maximum transmitted power $P_{T}$ of the secondary transmitter can be selected according to the rule

$$
P_{T}= \begin{cases}\frac{Q}{\|\mathbf{G}\|_{F}^{2}} & \|\mathbf{G}\|_{F}^{2}>\frac{Q}{P} \\ P & \|\mathbf{G}\|_{F}^{2} \leq \frac{Q}{P},\end{cases}
$$

where $\|\cdot\|_{F}^{2}$ is the squared Frobenius norm of the matrix and $P$ is the peak power of the secondary transmitter. As mentioned in [3], for $Q \gg P$ case, the effect of the interference temperature $Q$ disappears, which makes the performance analysis for the secondary system related to the peak power $P$ only and the analysis in that case reduces to that of the traditional MIMO system. Therefore, in this paper, like [2], we apply $P_{T}=Q \backslash\|\mathbf{G}\|_{F}^{2}$ as the total transmit power at the secondary transmitter. 
For notation simplicity, let $Y=\|\mathbf{G}\|_{F}^{2}$. Then, the received signal at the secondary receiver is given by

$$
\mathbf{y}=\sqrt{\frac{Q}{N Y}} \mathbf{H} \mathbf{s}+\mathbf{n}
$$

where $\mathbf{n}$ is the complex Gaussian noise with covariance matrix $\mathbf{I}_{N}$ and $\mathbf{s}$ denotes the transmit signal vector with covariance matrix $\mathbf{I}_{N}$.

\section{CAPACITY ANALYSIS}

In this section, we consider the capacity of a single-user SS MIMO system employing spatial multiplexing. Assuming the knowledge of CSI at the secondary transmitter, we provide closed-form capacity analysis for the optimal power allocation scheme and MIMO MRC systems.

A. Capacity Analysis for a System with Uniform Power Allocation

Based on the channel model (2), the instantaneous channel capacity between the secondary transmitter and its receiver can be written by

$$
C=\log _{2} \operatorname{det}\left(\mathbf{I}_{N}+\frac{Q}{N Y} \mathbf{H} \mathbf{H}^{\dagger}\right)
$$

where $\dagger$ denoted the transpose conjugate. Compared with the channel capacity of traditional MIMO system [4], the difference is the term $Y$. Thus, we can use some analytical techniques applied in the regular MIMO systems to evaluate the capacity (3). By using the singular value decomposition (SVD), (3) can be rewritten as

$$
C=\sum_{n=1}^{N} \log _{2}\left(1+\frac{Q}{N Y} \lambda_{n}\right)
$$

where $\lambda_{n}$ are the unordered nonnegative eigenvalues of $\mathbf{H H}^{\dagger}$ and we assume that $\lambda_{n}$ are independent of $Y$. From [6], the probability density function (PDF) of an unordered eigenvalue $\lambda_{n}$ is given by

$f_{\lambda_{n}}(\lambda)=\frac{1}{N} \sum_{i=0}^{N-1} \sum_{j=0}^{i} \sum_{l=0}^{2 j} \frac{(-1)^{l}(2 j) !}{2^{2 i-l} l !(j !)^{2}}\left(\begin{array}{c}2 i-2 j \\ i-j\end{array}\right)\left(\begin{array}{c}2 j \\ 2 j-l\end{array}\right) \lambda^{l} e^{-\lambda}$.

In flat Rayleigh fading channels, $Y$ is a sum of $N^{2}$ squared complex Gaussian random variables and follows the Chisquared distribution with $2 N^{2}$ degree of freedom. Thus, the PDF of $Y$ is readily given by [8]

$$
f_{Y}(y)=\frac{1}{\Gamma\left(N^{2}\right)} y^{N^{2}-1} e^{-y}
$$

With (5) and (6) at hand, the ergodic capacity between the secondary transmitter and the secondary receiver can be evaluated with the help of [9, Eq.(6.455.1)][10, Eq.(78)] as

$$
\begin{aligned}
E[C]= & \log _{2}(e) \sum_{i=0}^{N-1} \sum_{j=0}^{i} \sum_{l=0}^{2 j} \frac{(-1)^{l}(2 j) !}{2^{2 i-l} j ! j !}\left(\begin{array}{c}
2 i-2 j \\
i-j
\end{array}\right) \\
& \cdot\left(\begin{array}{c}
2 j \\
2 j-l
\end{array}\right) \sum_{s=1}^{l+1} \frac{1}{N^{2}+l+1-s} \\
& \cdot{ }_{2} F_{1}\left(1, N^{2} ; N^{2}+l+2-s ; 1-\frac{N}{Q}\right),
\end{aligned}
$$

where ${ }_{2} F_{1}(a, b ; c ; x)$ is the Gaussian hypergeometric function defined in [9].

For the special case when $N=1$ and with the help of [9, Eq.(9.121.6)], the capacity expression (7) reduces to

$$
E[C]=\frac{Q \log _{2}(Q)}{Q-1}
$$

which is in agreement with the previously known result [2, Eq.(24)]. Using the analyzing method in [11], $E[C]$ can be lower bounded as

$$
\begin{aligned}
E[C] \geq & N \log _{2}\left[1+Q \exp \left(\frac{1}{N} E\left\{\ln \operatorname{det}\left(\frac{\mathbf{H} \mathbf{H}^{\dagger}}{Y N}\right)\right\}\right)\right] \\
= & N \log _{2}\left[1+Q \exp \left(\frac{1}{N} E\left\{\ln \operatorname{det}\left(\mathbf{H H}^{\dagger}\right)\right\}\right.\right. \\
& -E\{\ln Y\})]
\end{aligned}
$$

From [11], we know

$$
E\left\{\ln \operatorname{det}\left(\mathbf{H H}^{\dagger}\right)\right\}=\sum_{n=1}^{N} \psi(N-n+1)-N \ln N
$$

and

$$
E\{\ln Y\}=\psi\left(N^{2}\right)
$$

where $\psi(\cdot)$ is the digamma function. Substituting (10) and (11) into (9) yields

$$
\begin{aligned}
E[C] \geq & N \log _{2}\left[1+Q \exp \left(\frac{1}{N} \sum_{n=1}^{N} \psi(N-n+1)\right.\right. \\
& \left.\left.-\ln N-\psi\left(N^{2}\right)\right)\right]
\end{aligned}
$$

For high $Q$, (12) reduces to

$$
\begin{aligned}
E[C] \approx & \log _{2}(e)\left[N \ln Q+\sum_{n=1}^{N} \psi(N-n+1)\right. \\
& \left.-N \ln N-N \psi\left(N^{2}\right)\right] .
\end{aligned}
$$

Although the SS MIMO system can still achieve the multiplexing gain $N$, we can see that the capacity of a SS MIMO system is less than that of the traditional MIMO system in comparison with the result in [11]. Note that in (13), $N \psi\left(N^{2}\right)$ accounts for the interference term. Also, from (13), we can see that the increase of the number of antennas may have a negative effect on the system capacity in the low signal-to-noise ratio (SNR) regime. The reason is that the $N \psi\left(N^{2}\right)$ term is dominant at low SNR in contrast to the $N \ln Q$ term. But with the increase of SNR, the channels with more antennas can provide higher 
capacity. This is unlike the traditional MIMO system case in which applying multiple antennas can yield a higher channel capacity in the low and high SNR regime cases.

Fig.2 shows the average capacity of SS MIMO systems when $N=1$ and 3 . The exact capacity and its lower bound are plotted by using (6) and (12), respectively. As expected, the simulation results perfectly match with their corresponding analysis. Also the lower bound is tight at high SNR. It is clearly observed that capacity of multiple antennas is less than that of the single-antenna case at low SNR regime. However at high SNR, the SS MIMO system provides higher capacity like it is the case for traditional MIMO systems.

\section{B. Optimal Adaptive Transmission}

From (4), we can see that the CR MIMO channels are decomposed into $N$ parallel sub-channels. Now assume that the transmitter can have access to the CSI on each sub-channel and that every sub-channel can adjust its transmit power $P_{i}$ according to the channel variations while satisfying the interference constraints $Y \sum_{i=1}^{N} P_{i}=Q$. As such, following the optimization problem formulated in [5], the achievable maximum capacity of the SS MIMO system under optimal power allocation can be written as

$$
\begin{gathered}
E[C]=\max N \int_{0}^{\infty} \int_{0}^{\infty} \log _{2}(1+P(\lambda, y) \lambda) f_{\lambda}(\lambda) f_{Y}(y) \mathrm{d} \lambda \mathrm{d} y \\
\text { subject to } N \int_{0}^{\infty} \int_{0}^{\infty} y P(\lambda, y) f_{\lambda}(\lambda) f_{Y}(y) \mathrm{d} \lambda \mathrm{d} y, \leq Q
\end{gathered}
$$

where $f_{\lambda}(\lambda)$ is the PDF of the unordered $\lambda_{n}$. Similar to [2], by using the Lagrangian method, the solution to the optimization problem (14) is a water-filling algorithm [12]

$$
P(\lambda, y)=\left[\frac{1}{\eta_{0} Y}-\frac{1}{\lambda}\right]^{+},
$$

where $\eta_{0}$ is the cutoff value and chosen to satisfy the power constraint as

$$
N \int_{0}^{\infty} \int_{\eta_{0} y}^{\infty} y\left(\frac{1}{\eta_{0} y}-\frac{1}{\lambda}\right) f_{\lambda}(\lambda) f_{Y}(y) \mathrm{d} \lambda \mathrm{d} y \leq Q .
$$

Substituting (5) and (6) into (16), we see that the cutoff value must satisfy

$$
\begin{aligned}
& \frac{\eta_{0}^{-\left(N^{2}+1\right)}}{\Gamma\left(N^{2}+2\right)} \sum_{i=0}^{N-1} \sum_{j=0}^{i} \sum_{l=0}^{2 j} \frac{(-1)^{l}(2 j) !}{2^{2 i-l} l !(j !)^{2}}\left(\begin{array}{c}
2 i-2 j \\
i-j
\end{array}\right)\left(\begin{array}{c}
2 j \\
2 j-l
\end{array}\right) \\
& \cdot \Gamma\left(N^{2}+l+1\right)_{2} F_{1}\left(N^{2}+l+1, N^{2} ; N^{2}+2 ;-\frac{1}{\eta_{0}}\right)=Q,
\end{aligned}
$$

where the integral tables [9, Eq.(6.455.1), Eq.(9.137.18), Eq.(9.131.1)] are used to obtain (17). From (17) with $N=1$, it can be easily verified that (17) reduces to [2, Eq.(12)]. Generally, numerical root finding technique is needed to obtain $\eta_{0}$. From (15), it is clearly observed that water-filling level is inversely proportional to the gain $Y$. For arbitrary sub-channel $n$, its cutoff value is $\eta_{0} Y$. In Fig.3, we plot the cutoff values for different number of antennas $N$. We can see that the cutoff value lies in the range [0,1]. Also, for a given $Q$, we can observe that the cutoff value is almost the same for all values of $N$. This is unlike the observation in [5] where the cutoff value is completely different for different numbers of antennas. The reason is that the cutoff value of $\lambda / Y$ is $\eta_{0}$ and all nodes are equipped with the same number of antennas $N$, which means that $\lambda$ increases and $Y$ also increases. This results in the variation of the ratio between $\lambda$ and $Y$ is not large.

Substituting (15) into (14) we obtain the capacity of a CR MIMO systems with optimal adaptive transmission as

$$
\begin{aligned}
E[C] & =\frac{\log _{2}(e)}{\Gamma\left(N^{2}\right)} \sum_{i=0}^{N-1} \sum_{j=0}^{i} \sum_{l=0}^{2 j} \frac{(-1)^{l}(2 j) !}{2^{2 i-l}(j !)^{2} l !}\left(\begin{array}{c}
2 i-2 j \\
i-j
\end{array}\right)\left(\begin{array}{c}
2 j \\
2 j-l
\end{array}\right) \\
& \times \Gamma\left(N^{2}+l+1\right) \int_{\eta_{0}}^{\infty} \ln \left(\frac{1}{\eta_{0}} x\right) \frac{x^{l}}{(x+1)^{N^{2}+l+1}} \mathrm{~d} x
\end{aligned}
$$

Unfortunately, no simple closed-form expression is available for the general case. However, when $N=1$ and using integration by part, (18) reduces to

$$
E[C]=\log _{2}\left(1+\frac{1}{\eta_{0}}\right),
$$

which is in agreement with the previously known result given in [2, Eq.(13)] for a single antenna spectrum sharing system.

With (8) and (19), we can investigate the capacity improvement induced by the optimal power and date adaptation for the $N=1$ case. Let us define the capacity difference as

$$
C_{d}=\log _{2}\left(1+\frac{1}{\eta_{0}}\right)-\frac{Q \log _{2}(Q)}{Q-1}=\log _{2} \frac{1+\frac{1}{\eta_{0}}}{Q^{\frac{Q}{Q-1}}}
$$

From [2, eq.12], we can see that $1 / \eta_{0}>Q$. Thus, for high $Q$, we have

$$
C_{d}>\log _{2} \frac{1+Q}{Q^{\frac{Q}{Q-1}}}=\log _{2} Q^{-\frac{1}{Q-1}}\left(1+\frac{1}{Q}\right) \rightarrow 0
$$

The above equation shows that the advantage of the waterfilling algorithm is negligible in the high SNR regime.

Fig.4 shows the capacity of SS MIMO systems under optimal or uniform power allocations strategies. Again, we can note the same observation as the ones made based on Fig.2. We note also that the optimal transmission scheme yields high capacity, as expected.

\section{SS MIMO Channels with Keyhole}

The analysis for the previous subsections is based on the assumption that the channels are i.i.d Rayleigh fading. However in some realistic wireless communication environments, the effect of keyhole channels may exist and degrade the channel capacity [6]. In this section, we consider a keyhole MIMO SS system where both of the links from the secondary transmitter to the primary receiver and the secondary receiver undergo the keyhole effect. According to the keyhole channels model [6][13], the capacity of a MIMO CR system with keyhole maybe can be expressed as

$$
C=\log _{2}\left(1+\frac{Q X_{1} X_{2}}{N Y_{1} Y_{2}}\right)
$$


where $X_{1}=\left\|\alpha_{1}\right\|^{2}, X_{2}=\left\|\beta_{1}\right\|^{2}, Y_{1}=\left\|\alpha_{2}\right\|^{2}$, and $Y_{2}=$ $\left\|\beta_{2}\right\|^{2}$. The vectors $\alpha_{i}$ and $\beta_{i}$ represent the scattering at the transmit and receive antenna arrays, respectively. For Rayleigh fading channels, these RVs all are Chi-square distributed with freedom of $2 N$. Following the analyzing method in [13], a lower bound for the average capacity can be written as

$E[C] \geq \log _{2}\left(1+\frac{Q}{N} \exp E\left\{\ln \frac{X_{1} X_{2}}{Y_{1} Y_{2}}\right\}\right)=\log _{2}\left(1+\frac{Q}{N}\right)$

Thus, it is interesting to observe that the SS MIMO system with keyhole channels on both channels and under the same number of antennas assumption can be lower bounded by the capacity of an AWGN channel.

In Fig.5, we plot the curves for the capacity of SS keyhole MIMO channels when $N=2,3$, and 4 . We can see that the lower bounds are very tight for all cases. Also, it is clearly observed that the capacity of such channels is completely inversely proportional to the number of antennas. Note that there is no diversity gain and no multiplexing gain for such kind of channels. This is unlike the traditional keyhole MIMO system for which a diversity gain can still be obtained [6].

\section{SS MIMO MRC System}

The above analysis focuses on a spatial multiplexing system. Now we turn to another transmission scheme, namely, the MIMO MRC. Such a scheme is applied at the secondary users. For the MIMO MRC scheme, both of the transmitter and the receiver are weighted by the weight vectors and the signals are combined in such a way that the SNR at the receiver combiner output is maximized. Such scheme requires the CSI is perfectly available at both the link ends.

Like [7], we can obtain the output SNR as

$$
\gamma=\frac{Q}{Y} \lambda
$$

where $\lambda$ is the largest eigenvalue of $\mathbf{H H}^{\dagger}$. The PDF of $\lambda$ for arbitrary numbers of transmit and receive antennas is given by [7]

$$
f(\lambda)=\sum_{k=1}^{N} \sum_{m=0}^{2 N k-2 k^{2}} d_{k, m} \frac{k^{m+1} \lambda^{m} e^{-k \lambda}}{m !}, \quad \lambda>0
$$

where the coefficients $d_{k, m}$ have been defined in [7].

With (24) and (25), the average capacity of an SS MIMO MRC system can be evaluated as

$$
\begin{aligned}
E[C] & =\sum_{k=1}^{N} \sum_{m=0}^{2 N-2 k^{2}} \sum_{s=1}^{m+1} \frac{d_{k, m} \log _{2}(e)}{N^{2}+m+1-s} \\
& \times{ }_{2} F_{1}\left(1, N^{2} ; N^{2}+m+2-s ; 1-\frac{k}{Q}\right) .
\end{aligned}
$$

Fig.6 shows the capacity comparison between the SS spatial multiplexing and the SS MIMO MRC system for $N=$ 2 , and 4. As expected, the simulations are in agreement with the analysis. It is observed that the MIMO MCR scheme has a little capacity improvement at low SNR in contrast to the spatial multiplexing scheme (7). Also, we can see that applying more antennas yields less capacity for the SS MIMO MRC system. This can be directly observed from (24). Although the maximal eigenvalue increase with the number of antennas, the ratio $Q / Y$ maybe decrease and in turn results in the degradation of capacity.

\section{CONCLUSIONS}

In this paper, we present a comprehensive analysis for spectrum sharing network with multiple antennas. More specifically, we derive some capacity expressions and use them to investigate the scaling law of SS MIMO systems under different transmission schemes and channel propagation environments. Results show that the SS MIMO systems operate well at high SNR regime. SS MIMO systems subject to the keyhole effect almost have little multiplexing gain and diversity gain. The capacity of MIMO MRC system is inversely proportional to the number of antennas over the whole SNR range.

\section{ACKNOWLEDGEMENT}

This publication was made possible by NPRP grant \#08152-2-043 and NPRP grant \#09-341-2-128 from the Qatar National Research Fund (a member of Qatar Foundation). The statements made herein are solely the responsibility of the authors.

\section{REFERENCES}

[1] V. Tarokh, New Directions in Wireless Communications Research Springer, 2009

[2] A. Ghasemi and E. S. Sousa, "Fundamental limits of spectrum-sharing in fading environments," IEEE Trans. Wireless Commun., vol.6, no.2, pp.649-658, Feb.2007.

[3] T. W. Ban, W. Choi, B. C. Jung and D. K. Sung, "Multiuser diversity in a spectrum sharing system," IEEE Trans. Wireless Commun., vol.8, no.1, pp.102-106, Jan.2009.

[4] G. J. Foschini, M. J. Gans, "On limits of wireless communications in a fading environment when using multiple antennas," Wireless Personal Commun., vol.6, pp.311-335, Mar.1998.

[5] S. K. Jayaweera, and H. V. Poor, "Capacity of multiple-antenna systems with both receiver and transmitter channel state information," IEEE Trans. Inform. Theory, vol.49, pp.2967-2709, Oct.2003.

[6] H. Shin and J. H. Lee, "Capacity of multiple-antenna fading channels:spatial fading correlation, double scattering, and keyholes," IEEE Trans. Inf. Theory, vol.49, pp.2636-2647, Oct.2003.

[7] P. A. Dighe, P. K. Mallik, S. S. Jamuar, "Analysis of transmit-receive diversity in Rayleigh fading," IEEE Trans. Commun., vol.51, pp.694-703, Apr.2003.

[8] J.G.Proakis, Digital Communications, 4rd ed. New York:McGraw-Hill, 2001.

[9] I. S. Gradshteyn and I. M. Ryzhik, Table of Integrals, Series, and Products, 6th ed. San Diego, CA:Academic, 2000.

[10] M. S. Alouini and A. J. Goldsmith, "Capacity of Rayleigh fading channels under different adaptive transmission and diversity-combining techniques," IEEE Trans. Veh. Technol., vol.48, pp.1165-1181, July 1999.

[11] Ö. Oyman, R. U. Nabar, H. Bölcskei, and A. J. Paulraj, "Characterizing the Statistical properties of mutual information in MIMO Channels,'IEEE Trans. Signa. Processing., vol.51, pp.2784-2795, Nov 2003.

[12] A. J. Goldsmith and P. P. Varaiya,"Capacity of fading channels with channel side information," IEEE Trans. Inf. Theory, vol.43,no.6,pp.19861992, MOv.1997.

[13] X. W. Cui and Z. M. Feng, "Lower capacity bound for MIMO correlated fading channels with keyhole,"IEEE Commun. Lett.,vol.8, no.8, pp.500502, Aug.2004. 


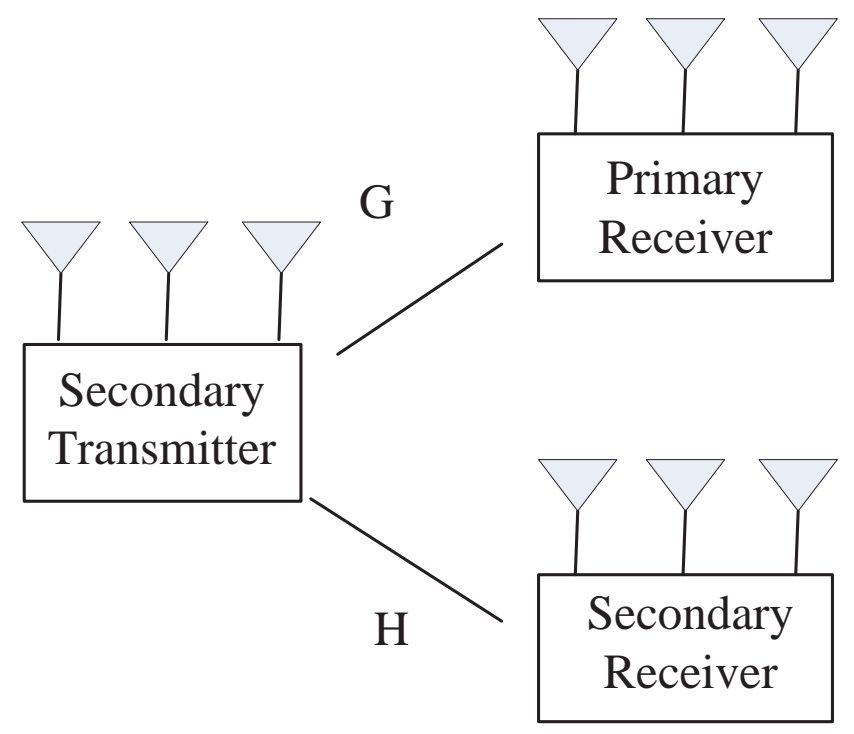

Fig. 1. Channel model of a SS MIMO system.

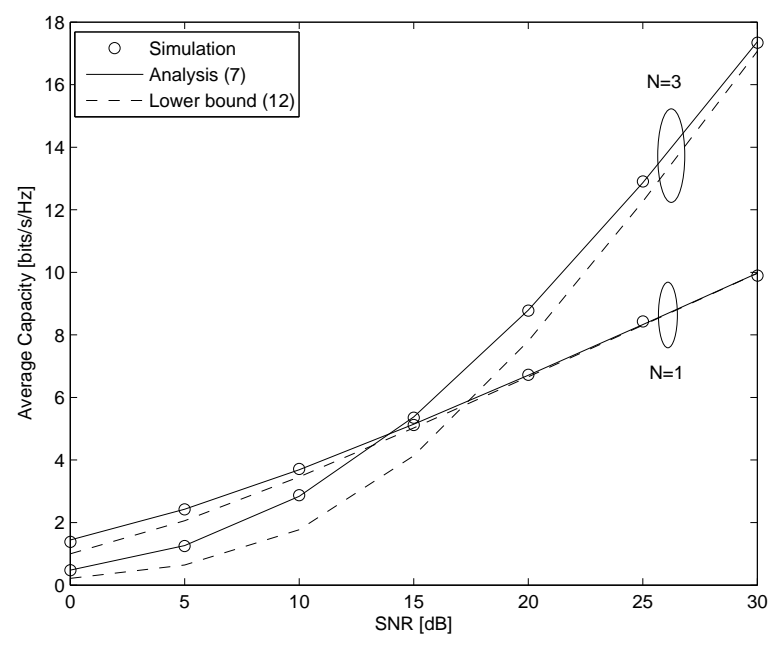

Fig. 2. Average capacity of SS MIMO systems.

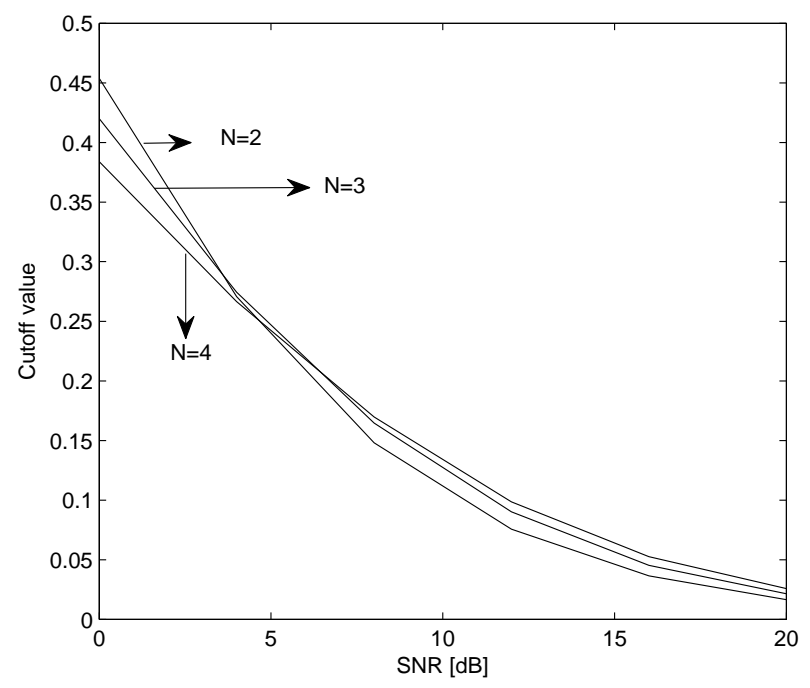

Fig. 3. Cutoff value for different numbers of antennas.

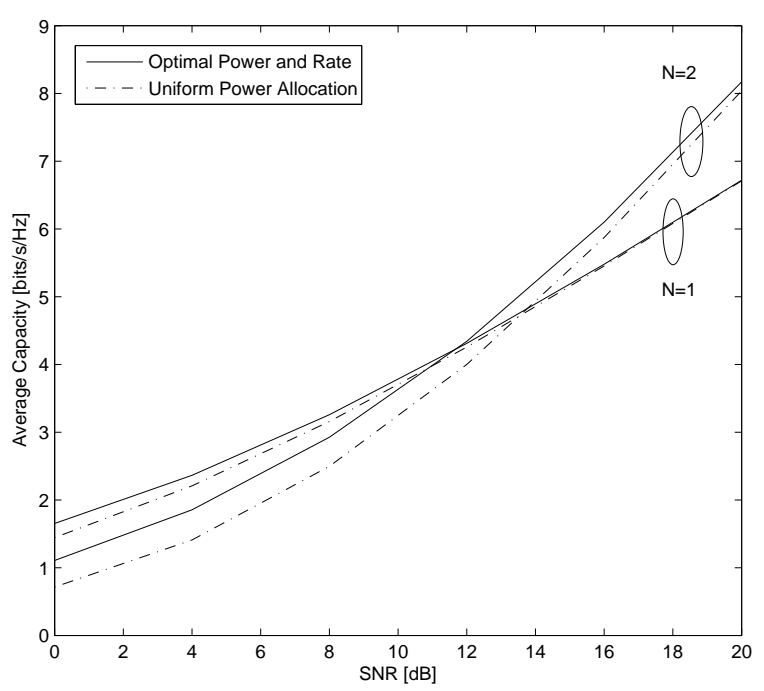

Fig. 4. Average capacity of SS MIMO systems with and without optimal power allocation.

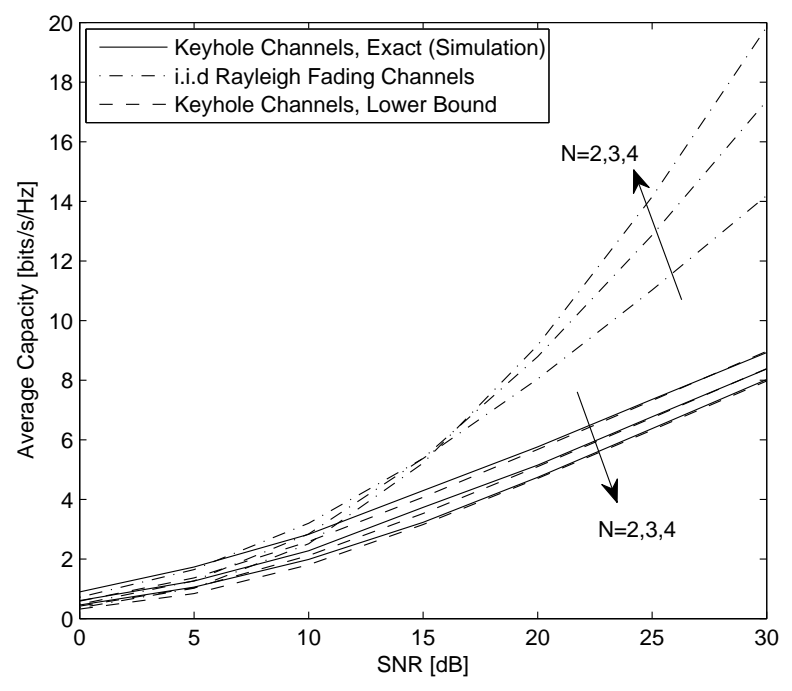

Fig. 5. Average capacity of SS MIMO systems subject to the keyhole effect.

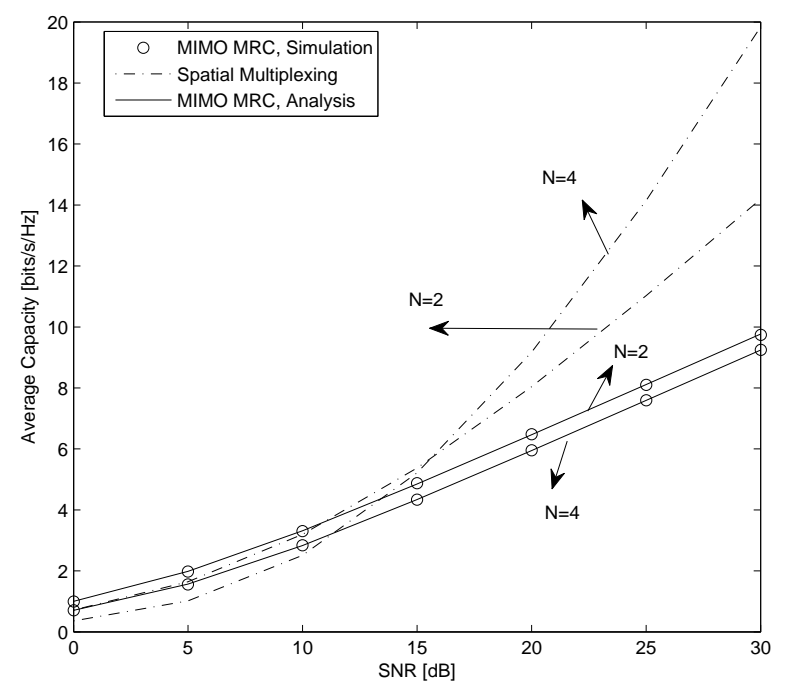

Fig. 6. Average capacity of SS MIMO MRC systems. 